\title{
Efectos de la rehabilitación pulmonar de corta duración en pacientes con EPOC
}

\author{
Effects of short pulmonary rehabilitation in patients with COPD
}

\author{
E. Paz Fernández, A. López García, L. González Doniz, S. Souto Camba, R. Fernández \\ Cervantes
}

\begin{abstract}
Resumen
Objetivo. Conocer los efectos sobre la tolerancia al ejercicio, disnea y calidad de vida en los programas de RP de corta duración en pacientes con EPOC.

Estrategias de búsqueda. Se realizaron búsquedas en Pubmed, PEDro y Cochrane de estudios con programas de rehabilitación pulmonar supervisados de corta duración, con intervención en músculos respiratorios y miembros inferiores.

Selección de estudios. Se consideraron 13 artículos, con programas de 10 a 18 sesiones, en un período de intervención de 3 a 9 semanas.

Síntesis de resultados. De los 13 estudios que midieron tolerancia al ejercicio, 9 mostraron resultados clínicamente significativos. Todos los estudios que valoraron la disnea presentaron mejoras en los valores finales. Dos estudios manifestaron significación clínica en todos los dominios de calidad de vida, 5 en la puntuación total del cuestionario y 5 en el dominio de síntomas.

Conclusiones. La RP de corta duración igual o superior a 16 sesiones muestra efectos positivos sobre la tolerancia al ejercicio, disnea y calidad de vida, con componentes mínimos de entrenamiento muscular de miembros superiores e inferiores y charlas de educación.
\end{abstract}

\begin{abstract}
Objective. To determine the effects on exercise tolerance, dyspnea, and quality of life in short pulmonary rehabilitation programs in patients with COPD.

Search strategies. We searched in Pubmed, PEDro and Cochrane studies of supervised programs of short pulmonary rehabilitation, with intervention in respiratory muscles and lower limbs.

Study selection. A total of 13 studies, with programs from 10 to 18 sessions are considered, in an intervention period of 3-9 weeks.

Synthesis results. A total of 9 of 13 studies that measured exercise tolerance showed clinically meaningful outcomes. All studies assessing dyspnea had improved the final values. Two studies showed clinical significance in all quality of life domains, 5 in the total score of the questionnaire and 5 in symptoms domain.

Conclusions. The short-term pulmonary rehabilitation programs less than 16 sessions shows positive effects on exercise tolerance, dyspnea and quality of life, with a minimum components of muscular training upper and lower limbs and education.
\end{abstract}

\section{Palabras clave}

Enfermedad pulmonar obstructiva crónica; Fisioterapia; Ejercicio

Keywords

Chronic obstructive pulmonary disease; Physical therapy modalities; Exercise 


\section{Introducción}

La enfermedad pulmonar obstructiva crónica (EPOC) es un proceso crónico y progresivo, caracterizado por la limitación al flujo aéreo de poca reversibilidad causada por una respuesta inflamatoria anormal, cuyo factor etiológico principal es el humo del tabaco ${ }^{1}$. Se trata de una enfermedad sistémica que, además de la disfunción pulmonar, consta de alteraciones cardiovasculares, músculoesqueléticas, nutricionales, anemia y depresión entre otras, provocando la merma del estado de salud y calidad de vida del individuo ${ }^{1,2}$ and 3 . En ella se produce una disfunción muscular, con anormalidades estructurales, funcionales y metabólicas, que condiciona una disminución de la tolerancia al ejercicio, dificultando la realización de actividad física y generando deterioro progresivo e incapacitante para el paciente $^{3 \text { and } 4}$.

Se estima que entre el 9,1 y el 10,2\% de la población adulta de más de 40 años padece EPOC, encontrándose una fuerte relación de estos valores con el hábito tabáquico (el 15\% de los fumadores activos y el $12,8 \%$ de exfumadores padecen EPOC) ${ }^{1 \text { and } 2}$. El ser fumador pasivo, la quema de combustibles biomasa, algunas profesiones o el déficit de $\alpha-1$ antitripsina aumentan las posibilidades de padecerla ${ }^{1}$.

Es la cuarta causa de muerte en los países occidentales, lo que nos sitúa ante un problema sociosanitario importante, ya que la Organización Mundial de la Salud (OMS) estima que actualmente 210 millones de personas en el mundo la padecen, de las cuales más de 2 millones se encuentran en España. Esto provoca un coste sanitario elevado, con una media entre 1.712 y 3.238 euros anuales ${ }^{1}$.

La Global initative for chronic obstructive pulmonary disease (GOLD) propone 4 componentes de abordaje en la EPOC: asesoramiento y monitorización de la enfermedad, reducción de los factores de riesgo, manejo de la EPOC estable y manejo de las exacerbaciones ${ }^{5}$. Dentro de esos componentes se encuentra la rehabilitación pulmonar (RP), que está definida como una intervención multidisciplinaria, global e individualizada, dirigida a la estabilización o reversión de las manifestaciones sistémicas de la enfermedad, y que reduce los costes sanitarios de la enfermedad ${ }^{6,7}$ and 8 . El abordaje se plantea con el fin de conseguir mejorar la tolerancia al ejercicio, la calidad de vida y la sintomatología, con disminución de las exacerbaciones y mortalidad de la enfermedad a largo plazo ${ }^{7}$.

En la actualidad, los programas de RP incluyen como componentes fundamentales la educación del paciente y la familia, la fisioterapia respiratoria (reeducación respiratoria y aclaramiento mucociliar) y las técnicas de entrenamiento al ejercicio ${ }^{9}$. Se exige un mínimo de 4 semanas $^{8 \text { and } 9}$ de intervención, aunque habitualmente se realizan 8 semanas o 20 sesiones (3-5 sesiones/semana) supervisadas, pudiendo alcanzar las 12 semanas $^{6}$ and 9

Se han establecido dichas pautas como idóneas para el tratamiento de RP, pero este planteamiento supone un coste muy elevado, lo que tiene como consecuencia el acceso de un número limitado de pacientes a este tipo de intervenciones ${ }^{9}$, por lo que es necesario conocer los beneficios de un programa de RP de menos de 20 sesiones, con el fin de establecer una duración mínima óptima que produzca cambios significativos en el individuo.

\section{Objetivos}

Objetivo principal

Conocer los efectos obtenidos tras un programa de RP de corta duración en pacientes con EPOC.

\section{Objetivos secundarios}

- Establecer la duración mínima necesaria dentro de los programas de RP, que manifieste mejoras clínicamente significativas en tolerancia al ejercicio, disnea y calidad de vida en pacientes con EPOC.

- Determinar los componentes necesarios dentro de un programa de RP de corta duración que permitan alcanzar beneficios significativos en pacientes con EPOC. 


\section{Material y métodos}

\section{Estrategias de búsqueda}

Se han realizado búsquedas en Pubmed, PEDro y la Biblioteca Cochrane Plus, entre los meses de noviembre de 2012 y febrero de 2013, mediante búsquedas avanzadas para poder incluir los criterios de búsqueda determinados y concretar los resultados.

Se procedió mediante la utilización de términos Medical Subject Headings (MeSH), los cuales han ido variando conforme hemos avanzado en las búsquedas, terminando con una combinación de términos de lenguaje MeSH y lenguaje convencional. Las palabras utilizadas han sido: Chronic obstructive pulmonary disease, Physical therapy modalities, Exercise atendiendo al MeSH; y Exercise program, Muscle training, Pulmonary rehabilitation en lenguaje convencional.

Se realizaron un total de 7 búsquedas en Pubmed, 2 en PEDro y una en Cochrane, de las cuales se obtuvieron 46 artículos que cumplían los criterios de búsqueda y resultaban de interés para el tema establecido. Solo 13 artículos del total fueron considerados por cumplir los criterios de inclusión y exclusión.

\section{Criterios de inclusión y exclusión}

Hemos seguido las siguientes estrategias de búsqueda para la inclusión de artículos:

1. Fechas de publicación: artículos comprendidos entre 2007 y la actualidad.

2. Tipo de artículo: revisiones sistemáticas, ensayos clínicos y estudios aleatorizados.

3. Idiomas: inglés y español.

4. Especies de estudio: humanos.

Conforme la evolución de las búsquedas y debido al bajo número de artículos encontrados, se han realizado búsquedas ampliando las fechas de publicación desde 2005 hasta la actualidad. También se recurrió a fuentes secundarias de información, mediante revisión de la bibliografía de artículos de interés: se encontraron 2 artículos que suponen una excepción a los criterios de búsqueda, con años de publicación de 2001 y 2003, se tuvieron en consideración por el cumplimiento estricto de los criterios de inclusión y exclusión.

Los criterios de inclusión han sido:

- Programas de RP de menos de 20 sesiones de duración (corta duración).

- Realización de las sesiones del programa de RP de forma supervisada.

- Entrenamiento mediante ejercicio de miembros inferiores y músculos respiratorios, como mínimo.

- Comprobación de mediciones de los parámetros iniciales y tras la finalización de las sesiones.

Los criterios de exclusión han sido:

- Estudios que no especifiquen el programa de entrenamiento a seguir.

- Ausencia de valoración de la tolerancia al ejercicio o calidad de vida.

- Realización de sesiones de entrenamiento no supervisadas sin valoración final.

\section{Variables e instrumentos a analizar}

En la revisión bibliográfica, no se realizará un análisis concreto de las variables demográficas, sino que se buscará el análisis de las variables específicas medidas en los estudios incluidos.

Las variables específicas a tener en cuenta son:

Tolerancia al ejercicio físico: Entenderemos como tolerancia al ejercicio físico a la capacidad para realizar actividad física sin limitación atribuida a factores pulmonares, cardíacos o musculares ${ }^{10}$,

En la mayoría de los estudios se valora mediante la prueba de 6 min marcha ( 6 minute walk test [6 MWT]), aunque en un estudio realizan la medición a través de una prueba de marcha de 3 min, y en otro de $12 \mathrm{~min}$. En el $6 \mathrm{MWT}$ el paciente realizará marcha durante $6 \mathrm{~min}$, en un pasillo de bajo tráfico, donde los pacientes serán instruidos y estimulados a caminar enérgicamente. Se realiza una medición de la distancia recorrida (en m), la saturación de oxígeno, la frecuencia cardíaca y la disnea. Las pruebas de 3 y 12 min serán iguales pero con la variación del tiempo ${ }^{11}$. Actualmente, se considera clínicamente significativo un aumento igual o superior a $35 \mathrm{~m}$ con respecto al valor inicial de la prueba ${ }^{12}$. 
El resto de estudios, excepto uno, efectuó la medición a través del Incremental shuttle walk test (ISWT) y del Shuttle walk test o prueba de marcha de esfuerzo, el Endurance shuttle walk test (ESWT). El ISWT determina la capacidad máxima de deambulación: los pacientes son instruidos para caminar durante tanto tiempo como sea posible a la velocidad dictada por una señal auditiva. La prueba se realiza en un pasillo cerrado con una longitud de $10 \mathrm{~m}$, con un cono en cada extremo para evitar cambios abruptos de dirección, donde la velocidad de marcha inicial se fija en $0,5 \mathrm{~m} / \mathrm{s}$ y se aumentan $0,17 \mathrm{~m} / \mathrm{s}$ en cada min hasta alcanzar un máximo limitado por la sintomatología, incentivando en todo momento a caminar durante el máximo tiempo posible. El ESWT se ejecuta en un recinto cerrado con terreno llano de $10 \mathrm{~m}$ de longitud, con un calentamiento previo de 1,5-2 min, para después realizar la prueba con una velocidad fijada correspondiente al $80 \%$ del pico de consumo máximo de oxígeno (predicho en un ISWT inicial), siendo instruidos para caminar durante tanto tiempo como sea posible a la velocidad dictada por una señal auditiva ${ }^{13}$

Para aquellos estudios donde se evaluó la tolerancia al ejercicio mediante un test incremental (ISWT), según lo establecido en un estudio de Singh et al., se considera clínicamente significativo un aumento de $48 \mathrm{~m}$ en la distancia recorrida ${ }^{14}$. En los que realizaron la medición de la tolerancia al ejercicio mediante el ESWT, se debe tener en consideración que los valores clínicamente significativos obtenidos en un estudio de Pepin et al. refieren una mejora de $85 \mathrm{~s}$ en la prueba ${ }^{15}$.

Un estudio realizó la medición con una prueba incremental en cicloergómetro. Según la normativa de la SEPAR para pruebas de ejercicio cardiopulmonar, los protocolos incrementales se efectúan durante un período relativamente corto, con incremento progresivo de la carga hasta llegar al límite de la tolerancia determinado por la sintomatología del individuo. Constan de 4 fases: fase de reposo previo al ejercicio, fase de ejercicio sin carga, incremento progresivo de la carga hasta el límite de tolerancia y fase de recuperación. La frecuencia de trabajo del cicloergómetro será aproximadamente de 60 ciclos/min, y la magnitud de incremento de la carga dependerá de las características del paciente, realizándose incrementos de 10 a $20 \mathrm{~W} / \mathrm{min}$ en sujetos sedentarios sanos ${ }^{16}$. No se han encontrado valores de referencia relacionados con esta prueba que establezcan una mejora clínica significativa tras un período de entrenamiento.

Disnea: Se definirá disnea como «sensación subjetiva de falta de aire o dificultad para respirar». En la EPOC se presenta cuando el daño pulmonar es importante, aparece de forma progresiva y es el síntoma más invalidante, que condiciona la calidad de vida de los pacientes ${ }^{17}$. La medición de esta en los estudios se realizó con la utilización de la Medical research council scale (escala MRC) y la escala modificada de Borg.

La escala modificada de Borg consta de 12 niveles numéricos de disnea, entre 0, que implica la ausencia de disnea, y 10 donde encontraríamos el valor de disnea máximo ${ }^{18}$.

La MRC consta de 5 grados de percepción de falta de aire que aumentan gradualmente desde 0 , donde solo se percibe ausencia de aire ante ejercicio vigoroso, hasta el grado 4 , donde la falta de aire se percibe en las actividades de la vida diaria ${ }^{19}$.

Calidad de vida: La calidad de vida es la percepción del individuo de su situación en la vida, dentro del contexto cultural y de valores en que vive, en relación con sus objetivos, expectativas, valores e intereses $^{20}$. En los estudios se midió mediante la utilización de 2 cuestionarios específicos, el Saint George's respiratory questionnaire (SGRQ) o el Chronic respiratory questionnaire (CRQ), y únicamente en una ocasión con el cuestionario genérico SF-36 (Medical outcomes survey short form-36).

El SGRQ está diseñado para medir el impacto de la enfermedad respiratoria en la calidad de vida relacionada con la salud y bienestar. Consta de 50 ítems que se pueden agregar en una puntuación global, y 3 subescalas de síntomas (disnea, tos y sibilancias), actividad e impacto de la enfermedad. La puntuación irá desde 0 (mejor puntuación) a 100 (peor puntuación). Las puntuaciones bajas indicarán mejor estado de salud ${ }^{21}$. Se considera clínicamente significativa una mejora de 4 puntos en el valor final del ítem ${ }^{22}$.

El CRQ se desarrolló para medir la calidad de vida de pacientes con EPOC. Contiene 20 preguntas que se dividen en 4 dominios: disnea, fatiga, función emocional y manejo de la enfermedad. Las respuestas se pueden calificar desde 1 punto (deterioro máximo), hasta 7 puntos (no hay deterioro), expresando la puntuación final como media de cada dominio y media global ${ }^{21}$. Los valores clínicamente significativos se producen con mejora mínima de 0,5 puntos en cada dimensión 22,23 and 24.

El SF-36 es un cuestionario genérico de calidad de vida, que consta de 36 ítems que miden estado funcional de salud y bienestar. Se compone de 8 dominios (función física, rol físico, dolor corporal, salud general, vitalidad, función social, función emocional y salud mental), que proporcionan 2 puntuaciones resumen de calidad de vida correspondiente a la salud física y mental, y también un dominio que valora el cambio producido en su salud de un año a otro. Las puntuaciones van de 0 a 100, siendo las puntuaciones bajas las correspondientes a un peor estado de salud, y las más altas al mejor ${ }^{25}$ and 26 . Vilagut et al. toman 
como valor de referencia un aumento de 0,4 puntos sobre los valores iniciales, lo que supondría un aumento de la puntuación total y la consiguiente mejora en la calidad de vida percibida por el individuo ${ }^{26}$.

\section{Resultados}

\section{Selección de estudios}

Los 13 artículos considerados $27,28,29,30,31,32,33,34,35,36,37,38$ and 39 por cumplir los criterios de inclusión y exclusión son ensayos clínicos o estudios aleatorizados controlados, con programas de RP supervisada entre 10 y 18 sesiones, de 3 a 9 semanas de intervención fisioterápica. forma:

Los 33 restantes no se tuvieron en consideración porque no cumplían los criterios de la siguiente

- 15 artículos tuvieron una duración igual o superior a 20 sesiones.

- 10 artículos solo realizaron entrenamiento de músculos respiratorios.

- 2 artículos no realizaron evaluación de los resultados al final de las sesiones, no pudiéndose observar los hallazgos inmediatos tras realizar el programa de RP.

- Una revisión sistemática incluyó programas con una duración superior a 20 sesiones.

- Un ensayo clínico realizó un programa domiciliario no supervisado, sin comprobación de la correcta ejecución de los ejercicios.

- 2 artículos no cumplían las fechas establecidas en los criterios de búsqueda porque se habían publicado en los años 1998 y 2000.

- 2 artículos realizaron un programa de combinación entre una terapia farmacológica específica junto con el programa de RP.

\section{Tolerancia al ejercicio físico}

Todos los estudios realizaron la medición de la tolerancia al ejercicio con los diferentes test presentados en material y métodos (tabla 1 ).

Tabla 1. Resultados de las mediciones correspondientes a tolerancia al ejercicio, disnea y calidad de vida

\begin{tabular}{|c|c|c|c|c|c|}
\hline Autor (año de publicación) & Medición & Instrumento & Valor inicial & Mejora & $\mathrm{p}$ \\
\hline \multirow[t]{2}{*}{ Clini et al. $(2001)^{27}$} & Tolerancia al ejercicio & Prueba increm. cicloerg. & 68 & 14 & $<0,05$ \\
\hline & Disnea & Escala Borg & 6,4 & 0,4 & $<0,05$ \\
\hline \multirow[t]{6}{*}{ Hui et al. $(2003)^{28}$} & Tolerancia al ejercicio & $6 \mathrm{MWT}$ & 333 & 90 & $<0,001$ \\
\hline & Disnea & Escala Borg & 5,38 & 0,22 & 0,5 \\
\hline & Calidad de vida & CRQ & Disnea: 4,36 & Disnea: 1,06 & $<0,001$ \\
\hline & & & Fatiga: 4,54 & Fatiga: 0,74 & $<0,005$ \\
\hline & & & Func. emoc.: 5,05 & Func. emoc.: 0,61 & $<0,001$ \\
\hline & & & Manejo enf.: 5,28 & Manejo enf.: 0,91 & $<0,001$ \\
\hline \multirow[t]{6}{*}{ Norweg et al. $(2005)^{29}$} & Tolerancia al ejercicio & $6 \mathrm{MWT}$ & 268,92 & 61,47 & NS \\
\hline & Calidad de vida & CRQ & Disnea: 4,22 & Disnea: 0 & NS \\
\hline & & & Fatiga: 4,04 & Fatiga: 0,2 & NS \\
\hline & & & Func. emoc.: 5,18 & Func. emoc.: 0,16 & NS \\
\hline & & & Manejo enf.: 5,75 & Manejo enf.: 0,3 & $<0,01$ \\
\hline & & & Total: 19,13 & Total: 0,82 & $<0,01$ \\
\hline \multirow[t]{6}{*}{ Montes de Oca et al. $(2005)^{30}$} & Tolerancia al ejercicio & $6 \mathrm{MWT}$ & 402 & 93 & $<0,01$ \\
\hline & Disnea & Escala Borg & 3,6 & 0,8 & NS \\
\hline & Calidad de vida & SGRQ & Síntomas: 44 & Síntomas: 18 & $<0,05$ \\
\hline & & & Actividad: 74 & Actividad: 22 & $<0,05$ \\
\hline & & & Impacto: 49 & Impacto: 15 & NS \\
\hline & & & Total: 50 & Total: 12 & $<0,05$ \\
\hline \multirow[t]{4}{*}{ Rossi et al. $(2005)^{31}$} & Tolerancia al ejercicio & $6 \mathrm{MWT}$ & 448 & 89,6 & $<0,001$ \\
\hline & Calidad de vida & SGRQ & Síntomas: 46 & Síntomas: 5,96 & $<0,001$ \\
\hline & & & Actividad: 49 & Actividad: 3,60 & $<0,025$ \\
\hline & & & Impacto: 21 & Impacto: 2,08 & 0,161 \\
\hline
\end{tabular}


Tabla 1. Resultados de las mediciones correspondientes a tolerancia al ejercicio, disnea y calidad de vida

\begin{tabular}{|c|c|c|c|c|c|}
\hline Autor (año de publicación) & Medición & Instrumento & Valor inicial & Mejora & $\mathrm{p}$ \\
\hline & & & Total: 34 & Total: 4,80 & $<0,001$ \\
\hline \multirow[t]{4}{*}{ Arnardóttir et al. $(2005)^{32}$} & Tolerancia al ejercicio & $12 \mathrm{MWT}$ & $\begin{array}{l}\text { Grupo A - } 854 \\
\text { Grupo B: } 811\end{array}$ & $\begin{array}{l}\text { Grupo A49 } \\
\text { Grupo B-8 }\end{array}$ & $\begin{array}{l}<0,05 \\
\mathrm{NS}\end{array}$ \\
\hline & Disnea & Escala Borg & $\begin{array}{l}\text { Grupo A: } 7,2 \\
\text { Grupo B: } 8,3\end{array}$ & $\begin{array}{l}\text { Grupo A-0,9 } \\
\text { Grupo B-0,1 }\end{array}$ & $\begin{array}{l}<0,05 \\
\mathrm{NS}\end{array}$ \\
\hline & $\begin{array}{l}\text { Calidad de vida } \\
\text { (grupo A) }\end{array}$ & SGRQ & $\begin{array}{l}\text { Síntomas: } 54,7 \\
\text { Actividad: } 63,0 \\
\text { Impacto: } 38,4 \\
\text { Total: } 48,5\end{array}$ & $\begin{array}{l}\text { Síntomas: } 4,8 \\
\text { Actividad: } 0,7 \\
\text { Impacto: } 4,2 \\
\text { Total: } 1,6\end{array}$ & $\begin{array}{l}\text { NS } \\
\text { NS } \\
\text { NS } \\
\text { NS }\end{array}$ \\
\hline & $\begin{array}{l}\text { Calidad vida } \\
\text { (Grupo B) }\end{array}$ & SGRQ & $\begin{array}{l}\text { Síntomas: } 51.1 \\
\text { Actividad: } 68,6 \\
\text { Impacto: } 39,0 \\
\text { Total: } 49,9\end{array}$ & $\begin{array}{l}\text { Síntomas: } 1,1 \\
\text { Actividad: } 3.6 \\
\text { Impacto: } 1,5 \\
\text { Total: } 2\end{array}$ & $\begin{array}{l}\text { NS } \\
\text { NS } \\
\text { NS } \\
\text { NS }\end{array}$ \\
\hline \multirow{2}{*}{$\begin{array}{l}\text { Sewell et al. } \\
\text { (RP 8 sesiones) }\end{array}$} & Tolerancia al ejercicio & ISWT & 196 & 56,9 & $\mathrm{NE}$ \\
\hline & Calidad de vida & CRQ & $\begin{array}{l}\text { Disnea: } 2,6 \\
\text { Fatiga: } 3,5 \\
\text { Func. emoc.: } 4,4 \\
\text { Manejo enf.: } 4,4\end{array}$ & $\begin{array}{l}\mathrm{NE} \\
\mathrm{NE} \\
\mathrm{NE} \\
\mathrm{NE}\end{array}$ & $\begin{array}{l}<0,05 \\
<0,05 \\
<0,05 \\
<0,05\end{array}$ \\
\hline \multirow{2}{*}{$\begin{array}{l}\text { Sewell et al. } \\
\text { (RP } 14 \text { sesiones) }\end{array}$} & Tolerancia al ejercicio & ISWT & 166,3 & 63,6 & $\mathrm{NE}$ \\
\hline & Calidad de vida & CRQ & $\begin{array}{l}\text { Disnea: } 2,8 \\
\text { Fatiga: } 3,5 \\
\text { Func. emoc.: } 4,2 \\
\text { Manejo enf.: } 4,3\end{array}$ & $\begin{array}{l}\mathrm{NE} \\
\mathrm{NE} \\
\mathrm{NE} \\
\mathrm{NE}\end{array}$ & $\begin{array}{l}\text { NS } \\
\text { NS } \\
\text { NS } \\
\text { NS }\end{array}$ \\
\hline Regiane Resqueti et al. $(2007)^{34}$ & $\begin{array}{l}\text { Tolerancia al ejercicio } \\
\text { Disnea } \\
\text { Calidad de vida }\end{array}$ & $\begin{array}{l}3 \text { MWT } \\
\text { Escala MRC } \\
\text { CRQ }\end{array}$ & $\begin{array}{l}178,6 \\
3,4 \\
\text { Disnea: } 3,2 \\
\text { Fatiga: } 4,1 \\
\text { Func. emoc.: 4,5 } \\
\text { Manejo enf.: } 5,1\end{array}$ & $\begin{array}{l}2,8 \\
0,3 \\
\text { Disnea: } 0,1 \\
\text { Fatiga: } 0,4 \\
\text { Func. emoc.: } 0 \\
\text { Manejo enf.: } 0,1\end{array}$ & $\begin{array}{l}\mathrm{NE} \\
0,14 \\
\mathrm{NE} \\
\mathrm{NE} \\
\mathrm{NE} \\
\mathrm{NE}\end{array}$ \\
\hline Von Leupoldt et al. $(2008)^{35}$ & $\begin{array}{l}\text { Tolerancia al ejercicio } \\
\text { Disnea }\end{array}$ & $\begin{array}{l}6 \text { MWT } \\
\text { Escala Borg }\end{array}$ & $\begin{array}{l}449 \\
3,2\end{array}$ & $\begin{array}{l}39 \\
0,5\end{array}$ & $\begin{array}{l}<0,001 \\
<0,001\end{array}$ \\
\hline Calidad vida & SF-36 & $\begin{array}{l}\text { Salud física: NE } \\
\text { Salud mental: NE }\end{array}$ & $\begin{array}{l}\text { Salud física: NE } \\
\text { Salud mental: NE }\end{array}$ & $\begin{array}{l}0,002 \\
0,046\end{array}$ & \\
\hline Du Moulin et al. $(2008)^{36}$ & $\begin{array}{l}\text { Tolerancia al ejercicio } \\
\text { Calidad de vida }\end{array}$ & $\begin{array}{l}6 \mathrm{MWT} \\
\text { CRQ }\end{array}$ & $\begin{array}{l}511,2 \\
\text { Disnea: 5,3 } \\
\text { Fatiga: 5,0 } \\
\text { Func. emoc.: 5,5 } \\
\text { Manejo enf.: 5,9 } \\
\text { Total: 5,4 }\end{array}$ & $\begin{array}{l}19,4 \\
\text { Disnea: } 0,5 \\
\text { Fatiga: } 0,3 \\
\text { Func. emoc.: } 0,1 \\
\text { Manejo enf.: } 0,3 \\
\text { Total: } 0,3\end{array}$ & $\begin{array}{l}0,019 \\
0,010 \\
0,009 \\
0,078 \\
0,799 \\
0,024\end{array}$ \\
\hline Riario-Sforza et al. $(2009)^{37}$ & Tolerancia al ejercicio & $6 \mathrm{MWT}$ & $\begin{array}{l}\text { GOLD I-355 } \\
\text { GOLD II - } 324,8 \\
\text { GOLD III - } 327,4 \\
\text { GOLD IV-180 }\end{array}$ & $\begin{array}{l}\text { GOLD I-63 } \\
\text { GOLD II - 68,5 } \\
\text { GOLD III - 89,1 } \\
\text { GOLD IV-130 }\end{array}$ & $\begin{array}{l}\text { NS } \\
<0,001 \\
<0,001 \\
<0,001\end{array}$ \\
\hline Linneberg et al. $(2012)^{38}$ & $\begin{array}{l}\text { Tolerancia al ejercicio } \\
\text { Calidad de vida }\end{array}$ & $\begin{array}{l}\text { ESWT } \\
\text { SGRQ }\end{array}$ & $\begin{array}{l}193 \\
\text { Total: 53,2 }\end{array}$ & $\begin{array}{l}761 \\
\text { Total: } 2,5\end{array}$ & $\begin{array}{l}<0,001 \\
0,591\end{array}$ \\
\hline \multirow[t]{2}{*}{ Egan et al. $(2012)^{39}$} & $\begin{array}{l}\text { Tolerancia al ejercicio } \\
\text { Disnea }\end{array}$ & $\begin{array}{l}6 \text { MWT } \\
\text { Escala MRC } \\
\text { Escala Borg }\end{array}$ & $\begin{array}{l}355,5 \\
2,3 \\
0,7\end{array}$ & $\begin{array}{l}44,4 \\
0,5 \\
0,5\end{array}$ & $\begin{array}{l}0,002 \\
0,05 \\
0,011\end{array}$ \\
\hline & Calidad de vida & SGRQ & $\begin{array}{l}\text { Síntomas: } 69,2 \\
\text { Fatiga: } 66,5 \\
\text { Impacto }^{6}: 46,6 \\
\text { Total: } 56,1\end{array}$ & $\begin{array}{l}\text { Síntomas: } 2,3 \\
\text { Actividad: } 7,5 \\
\text { Impacto }^{6}: 10,4 \\
\text { Total: } 9,1\end{array}$ & $\begin{array}{l}\text { NS } \\
\text { NS } \\
0,05 \\
0,001\end{array}$ \\
\hline
\end{tabular}

3 MWT: test de 3 min marcha (m); 6 MWT: test 6 min marcha (m); 12 MWT: test de 12 min marcha (m); CRQ: Chronic respiratory questionnaire; enf.: enfermedad; Escala MRC: escala Medical research council; ESWT: Endurance shuttle walk test (s); func. emoc.: función emocional; GOLD: Global initative for chronic obstructive lung disease; ISWT: Incremental shuttle walk test (m); NE: no especificado; NS: no significativo; Prueba increm. cicloerg.: prueba incremental con cicloergómetro (W); SF-36: cuestionario Short form 36; SGRQ: Saint George's respiratory questionnaire. 
Ocho de los programas hicieron la medición con el test de 6 min marcha ${ }^{28,29,30,31,35,36,37 \text { and } 39}, 2$ mediante variantes en la duración de este, uno de $3 \min ^{34}$ y otro de $12 \min ^{32}$. De estos 8,7 de ellos obtuvieron mejoras clínicamente significativas (superiores a $35 \mathrm{~m}$ ), entre los $39(\mathrm{p}<0,001)^{35}$ y $130 \mathrm{~m}$ $(\mathrm{p}<0,001)^{37}$, mientras que el restante no obtuvo significación clínica ni estadística, con una mejora de $19,4 \mathrm{~m}(\mathrm{p}=0,019)$ del valor final de la prueba ${ }^{36}$.

En las 2 variantes presentadas del $6 \mathrm{MWT}$, la prueba de 3 min marcha manifestó una mejora de $2,8 \mathrm{~m}^{34}$. La de $12 \mathrm{~min}$ obtuvo una mejora de $49 \mathrm{~m}(\mathrm{p}<0,05)$ en el grupo de intervención $\mathrm{A}$, y de $8 \mathrm{~m}$ en el grupo B (no significativa) $)^{32}$, manifestando en ambas variantes un aumento de las capacidades del paciente.

Dos programas de RP realizaron mediciones con las variables del Shuttle walk test. Uno realizó la medición con el ISWT, con un aumento de 63,6 m del valor final con respecto al inicial en el grupo de 14 sesiones, y de 56,9 en el grupo de 8 sesiones ${ }^{33}$. El otro programa obtuvo los resultados con el ESWT, con valores desde $193 \mathrm{~s}$ iniciales, a un aumento de $761 \mathrm{~s}$ en el valor final $(\mathrm{p}<0,001)^{38}$.

Solo uno de los estudios midió con la prueba incremental en cicloergómetro, consiguiendo una mejora de $14 \mathrm{~W}$ sobre el valor inicial $(\mathrm{p}<0,05)^{27}$.

\section{Disnea (tabla 1)}

La disnea se midió en 7 de los estudios al inicio y al final de la realización del programa de rehabilitación $^{27,28,30,32,34,35 \text { and } 39}$.

En la valoración se utilizó la escala MRC en 2 de ellos ${ }^{34}$ and 39 . Los valores medidos estaban comprendidos entre la dificultad para andar en llano a la misma velocidad que las demás personas de su edad (grado 2) y el parar para respirar después de andar unos $100 \mathrm{~m}$ o pocos min en terreno llano (grado 3).

La medición con la escala modificada de Borg se realizó en 5 de ellos $27,28,30,32$ and 35 , con un estudio que utilizó ambas escalas ${ }^{39}$. Los valores iniciales fueron desde disnea ligera (valor 2) a disnea entre muy intensa y casi máxima (valor 9).

En todos los casos se produjo una disminución del valor de disnea. Esta mejora se encuentra entre 0,1 (no significativo) y $0,9(\mathrm{p}<0,05)^{32}$ según las mediciones realizadas con la escala modificada de Borg, y $0,3(\mathrm{p}=0,14)^{34}$ a $0,5(\mathrm{p}=0,05)^{39}$ según los valores de la MRC, suponiendo un descenso de la disnea final con respecto a la inicial.

\section{Calidad de vida (tabla 1)}

La calidad de vida del paciente se ha medido con los cuestionarios SGRQ, CRQ y SF-36. Estos mostraron las variaciones de los diferentes ítems, manifestando los cambios según la puntuación total obtenida.

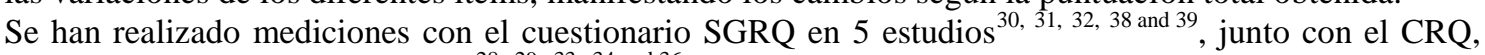

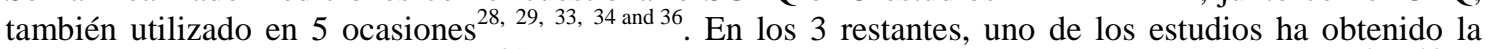
medición con el cuestionario SF-36 $6^{35}$, y 2 de ellos no han realizado mediciones de calidad de vida ${ }^{27}$ and 37. Los resultados obtenidos con el CRQ son los siguientes:

- Un estudio muestra mejora clínica y estadísticamente significativa de todos sus ítems. Este consta del aumento en la puntuación más elevado dentro de la variable disnea con 1,06 puntos $(\mathrm{p}<0,001)^{28}$.

- Otro obtuvo una mejora clínica y estadísticamente significativa en la puntuación total, con un aumento de $0,82(\mathrm{p}<0,01)$ del valor final con respecto al inicial ${ }^{29}$.

- Un estudio también tuvo un aumento clínica pero no estadísticamente significativo en el dominio disnea $^{36}$.

- De los 2 restantes, el programa de 8 sesiones tuvo una mejora estadísticamente significativa (no se pudo valorar la clínica), y el de 14 sesiones no obtuvo mejoras estadísticas significativas ${ }^{33}$. El otro estudio no tuvo significatividad clínica, y no se pudo valorar la estadística ${ }^{34}$.

En lo referente al SGRQ, los hallazgos fueron los siguientes:

- Un estudio mostró mejoras clínicamente significativas de todos $\operatorname{los}$ ítems ${ }^{30}$, siendo estadísticamente significativo el valor de los dominios excepto el de impacto.

En 2 ocasiones, se produjo una mejora clínica y estadísticamente significativa en la puntuación total $^{31 \text { and } 39}$ con los valores de 4,80 $(\mathrm{p}<0,001)^{31}$ y $9,1(\mathrm{p}<0,001)^{39}$.

- El dominio síntomas también presentó mejora clínica en 2 ocasiones ${ }^{31}$ and 32 , pero solo en una fue estadísticamente significativa ${ }^{31}$. 
- Los ítems actividad e impacto de la enfermedad fueron clínicamente significativos en una ocasión, con mejora estadísticamente significativa en el impacto $(\mathrm{p}<0,05)^{39}$.

- En el estudio restante ${ }^{38}$ solo se indicó la puntuación total del cuestionario, sin obtener un valor clínica o estadísticamente significativo.

El cuestionario SF-36 ${ }^{35}$ no presentó los valores iniciales y finales de las puntuaciones, impidiendo la valoración de la significatividad clínica. Estadísticamente, la mejora en salud física fue significativa.

\section{Discusión}

Los programas de corta duración considerados en esta revisión bibliográfica van desde las 8 sesiones ${ }^{33}$ hasta las 18 sesiones $^{30}$. Constan desde entrenamiento muscular hasta un programa multidisciplinario completo, con gran variabilidad en el abordaje de los programas.

\section{Tolerancia al ejercicio}

En todos los estudios se produjo una mejoría de los valores finales en las pruebas de tolerancia al ejercicio, superando el umbral clínicamente significativo en la prueba de marcha de 6 min (6 MWT) en 7 de las 8 ocasiones en las que se hizo la misma ${ }^{28,29,30,31,35,36,37 \text { and } 39}$, al igual que en las pruebas ISWT ${ }^{33} \mathrm{y}$ ESWT, en las que también se logró dicho aumento ${ }^{38}$.

En los estudios de Hui et al..$^{28}$ y Montes de Oca et al. ${ }^{30}$, cuyos programas de RP fueron similares y con una duración de 16 y 18 sesiones respectivamente, se muestra mejoría clínica y estadísticamente significativa en la tolerancia del ejercicio medida a través del 6 MWT (tabla 1). Se podría deducir que se pueden obtener logros en tolerancia al ejercicio con un programa de RP con una duración de 16 sesiones.

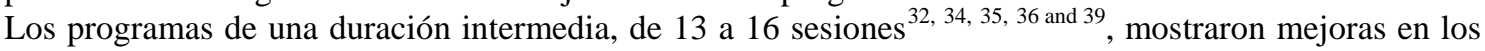
valores finales de las pruebas de tolerancia al ejercicio, que fueron clínicamente relevantes y estadísticamente significativos excepto en el estudio de Regiane Resqueti et al. ${ }^{34}$ (donde no se especificó la significación estadística, y los resultados del test no fueron clínicamente relevantes). El tamaño de la muestra utilizado puede estar influyendo en la no significación estadística; y la no relevancia clínica puede deberse a que la tolerancia al ejercicio inicial del grupo estudiado no estaba tan alterada, teniendo en cuenta los metros recorridos en la 6 MWT de base, y por tanto, el potencial de recuperación era menor con respecto a los que tienen más disminuida la capacidad de ejercicio. Esto se hace evidente en el estudio realizado por Riario-Sforza et al. ${ }^{37}$, donde los pacientes con mayor deterioro de la tolerancia al ejercicio fueron los que mayor recuperación obtuvieron al comparar diferentes grados de alteración (tabla 1).

En el estudio mencionado anteriormente ${ }^{37}$, se logró un aumento de $130 \mathrm{~m}$ recorridos con respecto al valor basal (tabla 1). Dicha mejora se produjo en un período de 10-12 sesiones. El protocolo constó de intervención multidisciplinar con entrenamiento muscular y educación para la enfermedad (tabla 2), y un elemento diferencial con respecto al resto de estudios fue el abordaje de agilidad de miembros superiores e inferiores, que unidos al abordaje multidisciplinar, son elementos que pueden haber favorecido los resultados obtenidos en la tolerancia al ejercicio.

Tabla 2. Resumen de la intervención en los programas de rehabilitación pulmonar

\begin{tabular}{lll}
\hline $\begin{array}{l}\text { Autor (año de } \\
\text { publicación) }\end{array}$ & N. ${ }^{\circ}$ de sesiones & Características de la rehabilitación pulmonar \\
\hline & & \\
Clini et al. $(2001)^{27}$ & $10-12$ sesiones & Optimización de tratamiento farmacológico \\
& Entrenamiento: \\
& - Ejercicio progresivo hasta alcanzar 30 min de ejercicio continuo \\
& - Fortalecimiento de musculatura abdominal, miembros superiores e inferiores mediante \\
& levantamiento de pesas \\
& - Cicloergómetro de miembros superiores \\
& Programa de nutrición \\
& Programa de asesoramiento psicosocial \\
& Ejercicio aeróbico incremental: marcha, bicicleta ergométrica \\
& & Ejercicio de fuerza de miembros superiores e inferiores con pesas
\end{tabular}


Tabla 2. Resumen de la intervención en los programas de rehabilitación pulmonar

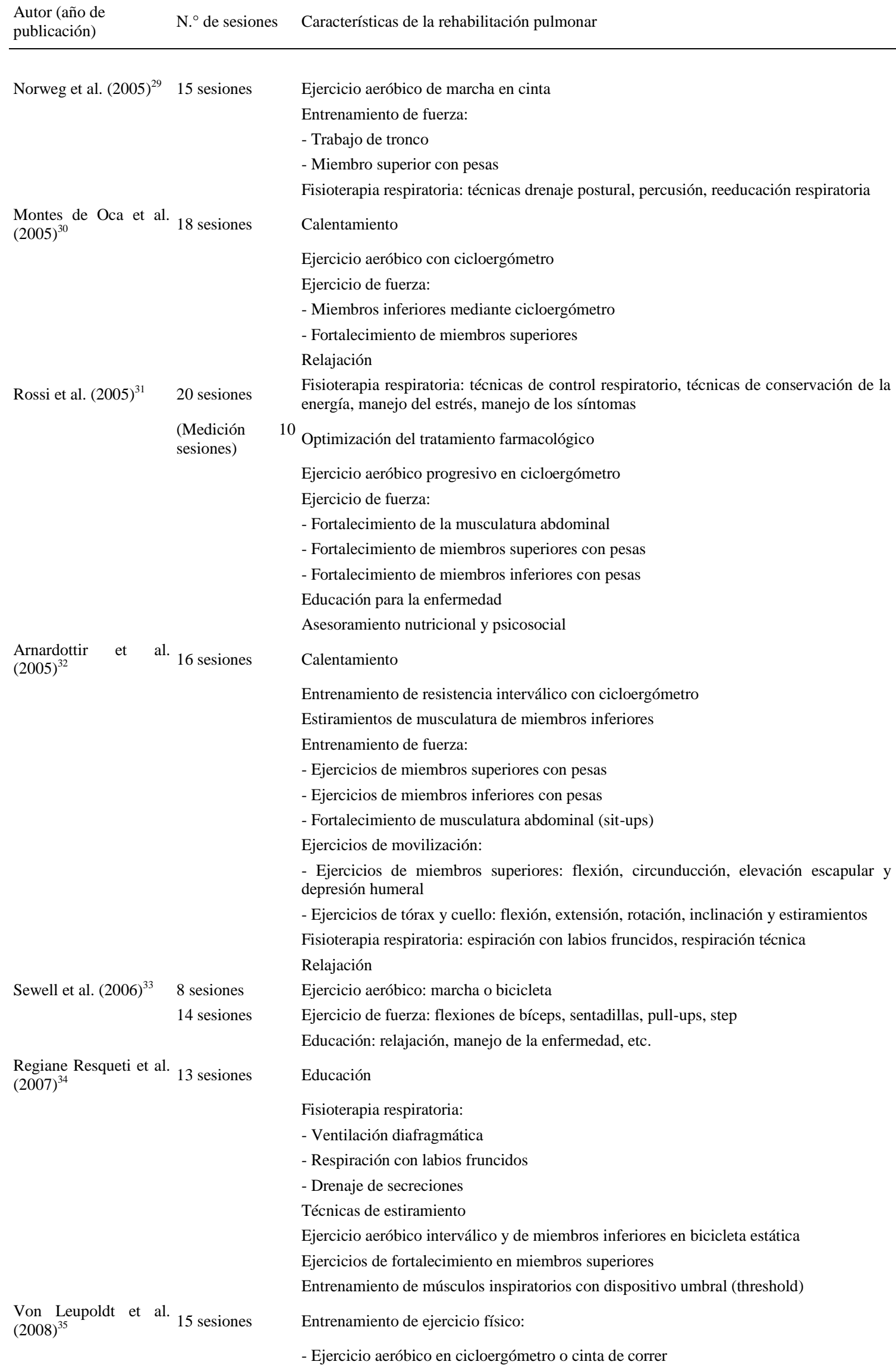

Norweg et al. $(2005)^{29} \quad 15$ sesiones

Montes de Oca et al. 18 sesiones $(2005)^{30}$

Rossi et al. $(2005)^{31} \quad 20$ sesiones

(Medición sesiones)

Arnardottir
$(2005)^{32}$ et al. 16 sesiones

Sewell et al. $(2006)^{33} \quad 8$ sesiones

14 sesiones

Regiane Resqueti et al. $(2007)^{34}$

13 sesiones

Von Leupoldt et al. 15 sesiones
$(2008)^{35}$

Ejercicio aeróbico de marcha en cinta

Entrenamiento de fuerza

- Trabajo de tronco

- Miembro superior con pesas

Fisioterapia respiratoria: técnicas drenaje postural, percusión, reeducación respiratoria

Calentamiento

Ejercicio aeróbico con cicloergómetro

Ejercicio de fuerza:

- Miembros inferiores mediante cicloergómetro

- Fortalecimiento de miembros superiores

Relajación

Fisioterapia respiratoria: técnicas de control respiratorio, técnicas de conservación de la energía, manejo del estrés, manejo de los síntomas

Optimización del tratamiento farmacológico

Ejercicio aeróbico progresivo en cicloergómetro

Ejercicio de fuerza:

- Fortalecimiento de la musculatura abdominal

- Fortalecimiento de miembros superiores con pesas

- Fortalecimiento de miembros inferiores con pesas

Educación para la enfermedad

Asesoramiento nutricional y psicosocial

Calentamiento

Entrenamiento de resistencia interválico con cicloergómetro

Estiramientos de musculatura de miembros inferiores

Entrenamiento de fuerza:

- Ejercicios de miembros superiores con pesas

- Ejercicios de miembros inferiores con pesas

- Fortalecimiento de musculatura abdominal (sit-ups)

Ejercicios de movilización:

- Ejercicios de miembros superiores: flexión, circunducción, elevación escapular y depresión humeral

- Ejercicios de tórax y cuello: flexión, extensión, rotación, inclinación y estiramientos Fisioterapia respiratoria: espiración con labios fruncidos, respiración técnica Relajación

Ejercicio aeróbico: marcha o bicicleta

Ejercicio de fuerza: flexiones de bíceps, sentadillas, pull-ups, step

Educación: relajación, manejo de la enfermedad, etc.

Educación

Fisioterapia respiratoria:

- Ventilación diafragmática

- Respiración con labios fruncidos

- Drenaje de secreciones

Técnicas de estiramiento

Ejercicio aeróbico interválico y de miembros inferiores en bicicleta estática

Ejercicios de fortalecimiento en miembros superiores

Entrenamiento de músculos inspiratorios con dispositivo umbral (threshold)

Entrenamiento de ejercicio físico:

- Ejercicio aeróbico en cicloergómetro o cinta de correr 
Tabla 2. Resumen de la intervención en los programas de rehabilitación pulmonar

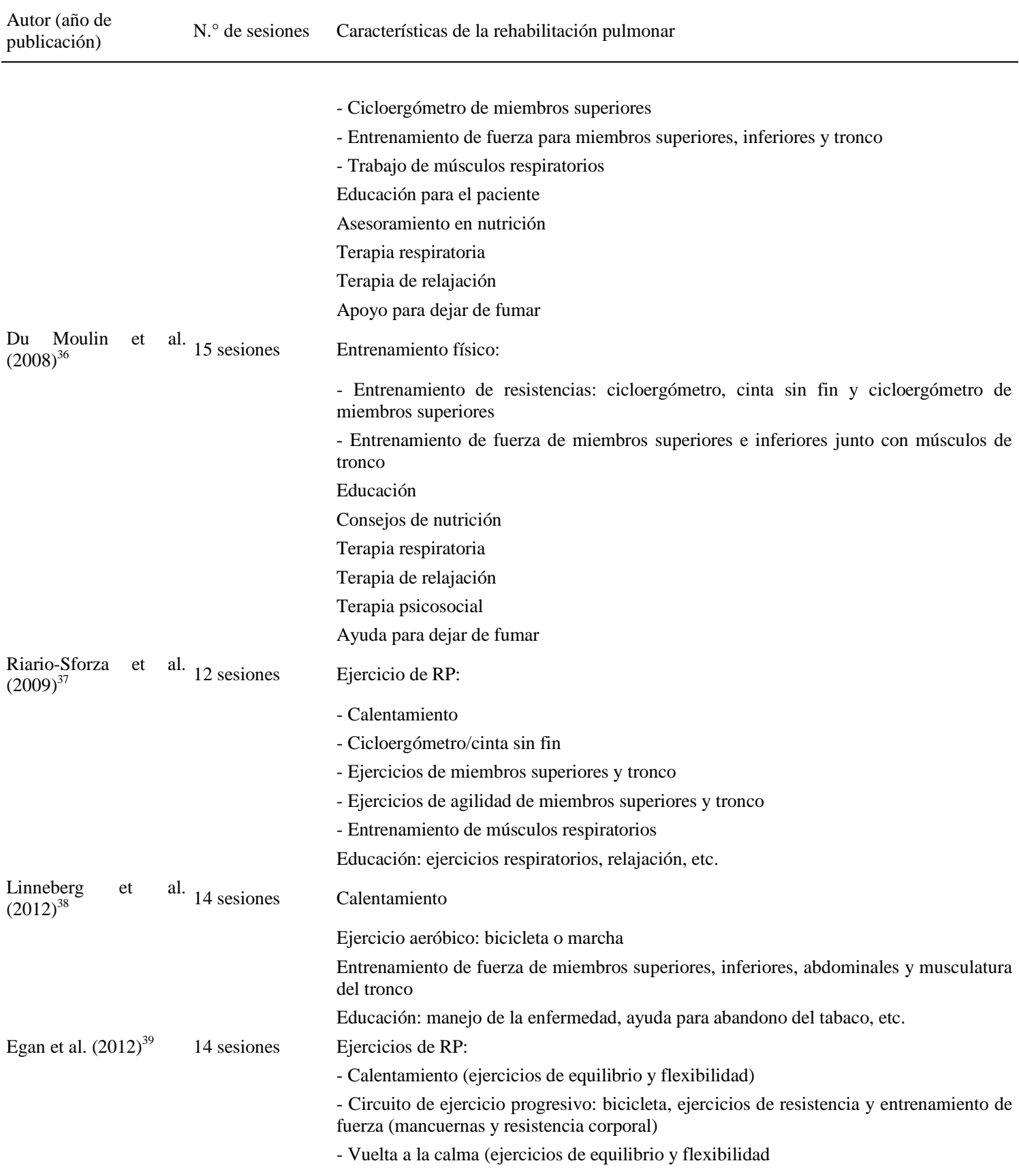

Comparando el estudio de Riario-Sforza ${ }^{37}$ con el de Rossi et al. ${ }^{31}$, los valores del primero ${ }^{37}$ son similares o inferiores a los del segundo ${ }^{31}$, cuyo programa de entrenamiento fue similar, pero además incluyó permeabilidad de la vía aérea (tabla 2). Se puede plantear que 10 sesiones tendrían un efecto similar sobre la tolerancia al ejercicio en pacientes con EPOC leve a severo, incluyendo un programa de RP con charlas informativas, pero 12 sesiones son más efectivas en pacientes de EPOC muy severo.

Los estudios de Sewell et al. ${ }^{33}$, Linneberg et al. ${ }^{38}$ y Clini et al. ${ }^{27}$ concuerdan con el anterior planteamiento. En el estudio realizado por Sewell et al. ${ }^{33}$, se compararon 2 grupos tratados con RP durante 8 y 14 sesiones. Los valores de tolerancia al ejercicio finales medidos con la prueba ISWT fueron ambos clínicamente significativos, con una diferencia de $6,7 \mathrm{~m}$ en el grupo que realizó 14 sesiones con respecto al grupo que realizó 8 sesiones. Con Linneberg et al. ${ }^{38}$, el ESWT también mostró una mejora que resultó ser clínica y estadísticamente significativa $(\mathrm{p}<0,001)$, en una intervención de 14 sesiones. En 
comparación con el programa de Rossi et al. ${ }^{31}$, los resultados sobre la tolerancia al ejercicio son similares entre ambos. Clini et al. ${ }^{27}$ realizaron una programa de RP de 10-12 sesiones, en el que la tolerancia al ejercicio se midió en una prueba incremental de cicloergómetro que mostró una mejora de $14 \mathrm{~W}$ con respecto al valor inicial, siendo dicho valor estadísticamente significativo $(\mathrm{p}<0,05)$. Este programa y el de Rossi et al. ${ }^{27}$ and 31 son similares en cuanto a sus componentes (tabla 2). Los 3 estudios anteriores incluían en los programas de intervención un protocolo de entrenamiento muscular, además, de tener un componente importante de educación. Lo cual podría ser uno de los factores que han influido en los logros obtenidos en la mejoría de la tolerancia al ejercicio en programas de RP con una duración entre 8 y 14 sesiones, aunque no se puede deducir qué protocolo de intervención es mejor, teniendo en cuenta que las medidas de tolerancia al ejercicio son heterogéneas.

Los resultados extraídos conforme a tolerancia al ejercicio nos indican que un programa de 10 sesiones supervisadas de RP muestra beneficios en programas multidisciplinarios que combinen la educación para el paciente con un entrenamiento físico completo (ejercicio aeróbico y entrenamiento de fuerza), para pacientes que se encuentren en estadios de leve a severo, mientras que los de 12 sesiones están más indicados en los que tengan EPOC muy severo.

\section{Disnea}

La disnea se midió en 7 de los 13 estudios considerados en esta revisión, mediante la escala

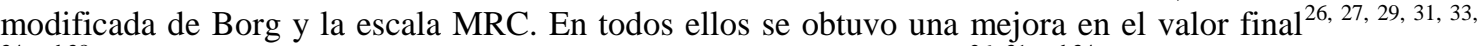
34 and 38 , pero solo en 3 de ellos fueron estadísticamente significativos ${ }^{26,31}$ and 34 , con medición mediante la escala modificada de Borg (tabla 1).

El mayor incremento se produjo en un estudio de Arnardottir ${ }^{32}$, con un aumento de 0,9 puntos (tabla 1), correspondiente a un programa de rehabilitación de 16 sesiones, con entrenamiento físico pero sin charlas educativas adicionales. Podemos considerar que un programa de RP de 16 sesiones sería el más efectivo para lograr una mejora estadísticamente significativa en la disnea.

\section{Calidad de vida}

En los estudios que evaluaron la calidad de vida se utilizaron cuestionarios específicos de enfermedad respiratoria (CRQ y SGRQ) en todas las ocasiones excepto en el de von Leupoldt et al. ${ }^{35}$, donde se ha utilizado un cuestionario genérico (SF-36).

Del total de estudios, Clini et al. ${ }^{27}$ y Riario-Sforza et al. ${ }^{37}$ no realizaron una valoración de la calidad de vida relacionada con la salud (CVRS). Dos estudios mostraron un aumento clínicamente significativo en todas las dimensiones (tabla 1$)^{28 \text { and } 30}$, con un número de sesiones de 16 y 18 respectivamente (tabla 2 ). En ellos se realizaba gran énfasis en el entrenamiento de musculatura periférica con cargas de trabajo altas, entre el 60 y $80 \%$ del valor inicial e involucrando musculatura de miembros superiores e inferiores. Este resultado concuerda con las observaciones realizadas en las guías de programas de RP aportadas por diferentes estamentos ${ }^{6}$, donde se refiere que el entrenamiento con cargas próximas al $80 \%$ de la máxima tolerada obtiene mejores resultados en los programas de RP.

El resto de programas mostraron mejoras clínica y estadísticamente significativas en la puntuación total del cuestionario. La duración de los programas correspondía a $10^{31}, 14^{39}$ y 15 sesiones $^{29}$. En el programa de 10 sesiones se observa un valor clínica y estadísticamente relevante en la dimensión de síntomas ${ }^{31}$, al contrario que en el de 14 sesiones, que fue el único dominio donde no obtuvo una mejora clínica significativa (tabla 1$)^{39}$. El programa de 15 sesiones solo muestra relevancia estadísticamente significativa en el ítem manejo de la enfermedad. En el estudio de Rossi et al. ${ }^{31}$, se realizó un abordaje multidisciplinario, a diferencia de los otros ensayos, lo que apunta a la gran importancia del manejo integral que se debe realizar en este tipo de pacientes, y más aún, si el programa es de corta duración.

Según los resultados obtenidos de esta revisión se puede apreciar que para lograr una mejora en la totalidad de las dimensiones de calidad de vida, en los programas de RP es necesaria una duración mínima de 16 sesiones, realizando entrenamiento de músculos periféricos. En la medida en que sean de más corta duración, aumenta la relevancia de un manejo multidisciplinario.

La evidencia científica demuestra los beneficios que los pacientes pulmonares crónicos obtienen con los programas de RP. No obstante este tipo de tratamiento no farmacológico genera un coste económico importante para el sistema de salud, lo cual se refleja en el número limitado de pacientes que se pueden beneficiar de este tipo intervenciones. Por lo tanto en la medida en que se logren resultados óptimos en las metas propuestas con programas de más corta duración que la estipulada actualmente, mayor número de pacientes van a poder acceder a este tratamiento. A pesar de esto hace falta mayor investigación sobre los 
parámetros específicos que deben tener los protocolos de entrenamiento muscular para lograr óptimos resultados.

Las limitaciones que se ha encontrado en la realización de este trabajo fueron la variabilidad de pruebas y unidades, que dificultó obtener resultados que permitieran realizar un buen análisis comparativo. Además algunos estudios no presentaban valores iniciales o finales de datos relevantes para poder efectuar una discusión coherente.

Con esta revisión se puede concluir que para lograr resultados en un programa de RP, en la tolerancia al ejercicio, calidad de vida y disnea, se necesita como mínimo un protocolo de entrenamiento muscular, con una intensidad entre el 60 y el $80 \%$ de la carga máxima inicial y con una duración de 16 sesiones. Y en la medida en que sean de más corta duración, aumenta la relevancia de un abordaje multidisciplinario y la importancia del componente de educación.

\section{Responsabilidades éticas}

\section{Protección de personas y animales}

Los autores declaran que para esta investigación no se han realizado experimentos en seres humanos ni en animales.

\section{Confidencialidad de los datos}

Los autores declaran que en este artículo no aparecen datos de pacientes.

\section{Derecho a la privacidad y consentimiento informado}

Los autores declaran que en este artículo no aparecen datos de pacientes.

\section{Conflicto de intereses}

Los autores declaran no tener ningún conflicto de intereses.

\section{Agradecimientos}

Agradecemos a todas aquellas personas que nos brindaron apoyo durante el trabajo que se ha realizado para este artículo, y a todos los que han colaborado o ayudado de alguna forma en este pequeño proyecto: sin vosotros no habría sido posible.

\section{Bibliografía}

1. Grupo de trabajo de GesEPOC. Guía práctica clínica para el diagnóstico y tratamiento de pacientes con enfermedad pulmonar obstructiva crónica (EPOC)-Guías Española de la EPOC (GesEPOC). Arch Bronconeumol., 48 (2012), pp. 2-58.

2. M.A. Llauger, M.A. Pou, L. Dominguez, M. Freixas, P. Valverde, C. Valero. Atención a la EPOC en el abordaje al paciente crónico en atención primaria. Arch Bronconeumol., 47 (2011), pp. 561-570.

3. B. Seoane, A. Mena, F. de la Iglesia. La enfermedad pulmonar obstructiva crónica y el internista - $1^{\mathrm{a}}$ parte: una enfermedad sistémica. Galicia Clín., 70 (2009), pp. 25-28.

4. C. Casanova, J.P. de Torres, M. Montes de Oca. Aspectos sistémicos y factores pronósticos. Arch Bronconeumol., 3 (2007), pp. 25-34.

5. K.F. Rabe, S. Hurd, A. Anzueto, P.J. Barnes, S.A. Buist, P. Calverley, et al. Global strategy for the diagnosis, management, and prevention of chronic obstructive pulmonary disease: GOLD executive summary. Am J Respir Crit Care Med., 176 (2007), pp. 532-555.

6. M.R. Guell, P. Cejudo, G. Rodriguez-Trigo, J.B. Galdiz, V. Casolive, M. Regueiro, et al. Standards for quality care in respiratory rehabilitation in patients with chronic pulmonary disease. Quality Healthcare Committee. Spanish Society of Pneumology and Thoracic Surgery (SEPAR). Arch Bronconeumol., 48 (2012), pp. 396-404.

7. F.C. Wehrmeister, M. Knorst, J.R. Jardim, E.C. Macedo, R.B. Noal, J. Martinez-Mesa, et al. Pulmonary rehabilitation programs for patients with COPD. J Bras Pneumol., 37 (2011), pp. 544-555.

8. Y. Lacasse, S. Martin, T.J. Lasserson, R.S. Goldstein. Meta-analysis of respiratory rehabilitation in chronic obstructive pulmonary disease. Eura Medicophys., 43 (2007), pp. 475-485

9. M.P. De Lucas, R. Güell, V. Sobradillo, C. Jiménez, M. Sangenis, T. Montemayor, et al. Rehabilitación respiratoria. Arch Bronconeumol., 36 (2000), pp. 34-43.

10. L. Bustamamente, M. Sívori, D. Lavagnino, A. Martínez, C. Sáenz. Discriminación de la limitación al ejercicio en pacientes con EPOC severa en pruebas máximas y submáximas. Rev Am Med Resp., 1 (2011), pp. 18-23.

11. M. Montes de Oca, M. Ortega, J. Lezama, J.M. Lopez. [Chronic obstructive pulmonary disease: Evaluation of exercise tolerance using three different exercise tests] [español]. Arch Bronconeumol., 37 (2001), pp. 69-74. 
12. M.A. Puhan, M.J. Mador, U. Held, R. Goldstein, G.H. Guyatt, H.J. Schunemann. Interpretation of treatment changes in 6-minute walk distance in patients with COPD. Eur Respir J., 32 (2008), pp. 637-643.

13. C. Brouillard, V. Pepin, J. Milot, Y. Lacasse, F. Maltais. Endurance shuttle walking test: Responsiveness to salmeterol in COPD. Eur Respir J., 31 (2008), pp. 579-584.

14. S.J. Singh, P.W. Jones, R. Evans, M.D. Morgan. Minimum clinically important improvement for the incremental shuttle walking test. Thorax., 63 (2008), pp. 775-777.

15. V. Pepin, L. Laviolette, C. Brouillard, L. Sewell, S.J. Singh, S.M. Revill, et al. Significance of changes in endurance shuttle walking performance. Thorax., 66 (2011), pp. 115-120.

16. Grupo de Trabajo de la SEPAR. Pruebas de ejercicio cardiopulmonar. Arch Bronconeumol., 37 (2001), pp. $247-$ 268.

17. C. Casanova, I. García-Talavera, J.P. de Torres. La disnea en la EPOC. Arch Bronconeumol., 41 (2005), pp. $24-$ 32.

18. G. Borg. Simple rating methods for estimation of perceived exertion. G. Borg (Ed.), Physical work and effort, Pergamon Press, Oxford (1977).

19. J.C. Bestall, E.A. Paul, R. Garrod, R. Garnham, P.W. Jones, J.A. Wedzicha. Usefulness of the Medical research council (MRC) dyspnoea scale as a measure of disability in patients with chronic obstructive pulmonary disease. Thorax., 54 (1999), pp. 581-586.

20. W.H.O.Q.O.L. Group. Study protocol for the World Health Organization Project to develop a quality of life assessment instrument (WHOQOL). Qual Life Res., 2 (1993), pp. 153-159.

21. M. Rutten-van, B. Roos, J.A. van Noord. An empirical comparison of the St George's respiratory questionnaire (SGRQ) and the Chronic respiratory disease questionnaire (CRQ) in a clinical trial setting. Thorax., 54 (1999), pp. 995-1003.

22. T. Hajiro, K. Nishimura. Minimal clinically significant difference in health status: The thorny path of health status measures?. Eur Respir J., 19 (2002), pp. 390-391.

23. J.E. Williams, S.J. Singh, L. Sewell, M.D. Morgan. Health status measurement: Sensitivity of the self-reported Chronic respiratory questionnaire (CRQ-SR) in pulmonary rehabilitation. Thorax., 58 (2003), pp. 515-518.

24. H.J. Schunemann, E.A. Akl, G.H. Guyatt. Interpreting the results of patient reported outcome measures in clinical trials: The clinician's perspective. Health Qual Life Outcomes., 4 (2006), p. 62.

25. J.J. Swigris, K.K. Brown, J. Behr, R.M. du Bois, T.E. King, G. Raghu, et al. The SF-36 and SGRQ: Validity and first look at minimum important differences in IPF. Respir Med., 104 (2010), pp. 296-304.

26. G. Vilagut, M. Ferrer, L. Rajmil, P. Rebollo, G. Permanyer-Miralda, J.M. Quintana, et al. El Cuestionario de salud SF-36 español: una década de experiencia y nuevos desarrollos. Gac Sanit., 19 (2005), pp. 135-150.

27. E. Clini, K. Foglio, L. Bianchi, R. Porta, M. Vitacca, N. Ambrosino. In-hospital short-term training program for patients with chronic airway obstruction. Chest., 120 (2001), pp. 1500-1505.

28. K.P. Hui, A.B. Hewitt. A simple pulmonary rehabilitation program improves health outcomes and reduces hospital utilization in patients with COPD. Chest., 124 (2003), pp. 94-97.

29. A.M. Norweg, J. Whiteson, R. Malgady, A. Mola, M. Rey. The effectiveness of different combinations of pulmonary rehabilitation program components: A randomized controlled trial. Chest., 128 (2005), pp. 663-672.

30. M. Montes de Oca, S.H. Torres, Y. Gonzalez, E. Romero, N. Hernandez, C. Talamo. [Changes in exercise tolerance, health related quality of life, and peripheral muscle characteristics of chronic obstructive pulmonary disease patients after 6 weeks' training] [español]. Arch Bronconeumol., 41 (2005), pp. 413-418.

31. G. Rossi, F. Florini, M. Romagnoli, T. Bellantone, S. Lucic, D. Lugli, et al. Length and clinical effectiveness of pulmonary rehabilitation in outpatients with chronic airway obstruction. Chest., 127 (2005), pp. 105-109.

32. R.H. Arnardottir, S. Sorensen, I. Ringqvist, K. Larsson. Two different training programmes for patients with COPD: A randomised study with 1-year follow-up. Respir Med., 100 (2006), pp. 130-139.

33. L. Sewell, S.J. Singh, J.E. Williams, R. Collier, M.D. Morgan. How long should outpatient pulmonary rehabilitation be? A randomised controlled trial of 4 weeks versus 7 weeks. Thorax., 61 (2006), pp. 767-771.

34. V. Regiane Resqueti, A. Gorostiza, J.B. Galdiz, E. Lopez, P. Casan, R. Guell. [Benefits of a home-based pulmonary rehabilitation program for patients with severe chronic obstructive pulmonary disease] [español]. Arch Bronconeumol., 43 (2007), pp. 599-604.

35. A. Von Leupoldt, E. Hahn, K. Taube, S. Schubert-Heukeshoven, H. Magnussen, B. Dahme. Effects of 3-week outpatient pulmonary rehabilitation on exercise capacity: Dyspnea, and quality of life in COPD. Lung., 186 (2008), pp. 387-391.

36. M. Du Moulin, K. Taube, K. Wegscheider, M. Behnke, H. van den Bussche. Home-based exercise training as maintenance after outpatient pulmonary rehabilitation. Respiration., 77 (2009), pp. 139-145.

37. G.G. Riario-Sforza, C. Incorvaia, F. Paterniti, L. Pessina, R. Caligiuri, C. Pravettoni, et al. Effects of pulmonary rehabilitation on exercise capacity in patients with COPD: A number needed to treat study. Int J Chron Obstruct Pulmon Dis., 4 (2009), pp. 315-319.

38. A. Linneberg, M. Rasmussen, T.F. Buch, A. Wester, L. Malm, G. Fannikke, et al. A randomised study of the effects of supplemental exercise sessions after a 7-week chronic obstructive pulmonary disease rehabilitation program. Clin Respir J., 6 (2012), pp. 112-119.

39. C. Egan, B.M. Deering, C. Blake, B.M. Fullen, N.M. McCormack, M.A. Spruit, et al. Short term and long term effects of pulmonary rehabilitation on physical activity in COPD. Respir Med., 106 (2012), pp. 1671-1679. 